\title{
Redefining Hypertension
}

Julieta Díaz-Juárez ${ }^{1}$ and Jorge Suarez ${ }^{2^{*}}$

${ }^{1}$ Departamento de Atención a la Salud. Universidad Autónoma Metropolitana Xochimilco, Ciudad de México, México

${ }^{2}$ Department of Medicine, University of California, San Diego, USA

"Corresponding author: Jorge Suarez, Research Scientist, Department of Medicine, 5063 Biomedical Sciences Building, University of California, San Diego, La Jolla, California, USA, Tel: 858-534-9931; E-mail: jsuarez@ucsd.edu

Received date: December 22, 2017; Accepted date: January 17, 2018; Published date: January 22, 2018

Copyright: (c) 2018 Juarez JD, et al. This is an open-access article distributed under the terms of the creative commons attribution license, which permits unrestricted use, distribution, and reproduction in any medium, provided the original author and source are credited.

\section{Editorial}

High blood pressure (hypertension) is associated with increased risk for cardiovascular disease (CVD), renal disease, atherosclerosis and all-cause mortality. Currently, the definition of hypertension in the US is based on systolic and diastolic blood pressure (BP) values that were established by the Joint National Committee on Prevention, Detection, Evaluation and Treatment of High Blood Pressure (JNC7) guidelines in 2003 [1]. Just recently, The American College of Cardiology and the American Heart Association (ACC/AHA) released the 2017 ACC/AHA/AAPA/ABC/ACPM/AGS/APhA/ASH/ASPC/NMA/PCNA Guideline for the Prevention, Detection, Evaluation, and Management of High Blood Pressure in Adults (2017 ACC/AHA guideline) [2]. The changes in the values to define hypertension in the new guideline have dramatic implications. Table 1 shows the new definitions and the systolic and diastolic values.

\begin{tabular}{|c|c|c|c|}
\hline Category & \multicolumn{2}{|c|}{ Systolic BP } & Diastolic BP \\
\hline Normal & $<120$ & and & $<80$ \\
\hline Elevated & $120-129$ & and & $<80$ \\
\hline Hypertension Stage 1 & $130-139$ & or & $80-89$ \\
\hline Hypertension Stage 2 & $\geq 140$ & or & $\geq 90$ \\
\hline Hypertension Crisis & $>180$ & and/or & $>120$ \\
\hline
\end{tabular}

Table 1: Definition of hypertension by the 2017 ACC/AHA guideline.

The most important changes are:

- Hypertension is now defined as readings of $130 \mathrm{~mm} \mathrm{Hg}$ and higher for the systolic blood pressure value, or readings of 80 and higher for the diastolic level. That is different from the previous definition of 140/90 and higher, reflecting complications that can occur at those lower numbers.

- In the first update to comprehensive U.S. guidelines on blood pressure detection and treatment since 2003, the category of prehypertension is removed.

- They predict that 14 percent more people will be diagnosed with high blood pressure and counseled to change lifestyle, however, there will only be a small increase in those who will be prescribed medication.

- The main idea is that by lowering the definition of hypertension, the guidelines recommend earlier intervention to prevent further increases in blood pressure and the complications.
The main goal of the new guidelines is to reduce de CVD risk associated with hypertension. The potential impact of adopting these guidelines has been analyzed $[3,4]$.

Khera et al., analyzed the impact of the new guidelines in the US and China concluding that there would be a $26.8 \%$ and $45.1 \%$ increase in those labeled with hypertension in the US and China, respectively. Furthermore, 7.5 million and 55.3 million will be newly recommended for therapy, and 13.9 million and 30 million newly recommended for intensification of existing therapy in the US and China, respectively [3].

These big numbers of potentially affected people give an idea of the dramatic impact that the new guidelines will have at all levels of the health care system including economic impact.

Regarding prevalence of metabolic syndrome (MetS) it will be also affected. More people will have the criteria to be diagnosed with MetS. Analysis of the impact of the new guidelines on MetS definition and impact must be performed as well as new strategies to treat the disease earlier than we do with the current criteria.

The main point of the changes is to start early treatment to prevent complications, however, the impact of early use of anti-hypertensive and other drugs has not been evaluated. All drugs have side effects that need to be considered. We will have to wait several years to see the results of these new guidelines and evaluate if the benefits are worth the cost and life style changes as well as the possible side effects caused by the drugs for people that was considered healthy before and now is hypertensive.

\section{References}

1. Chobanian AV, Bakris GL, Black HR (2003) The seventh report of the joint national committee on prevention, detection, evaluation, and treatment of high blood pressure: The jnc 7 report. JAMA 289: 2560-2571.

2. Whelton PK, Carey RM, Aronow WS, Casey DE, Collins KJ, et al. (2017) 2017 ACC/AHA/AAPA/ABC/ACPM/AGS/APhA/ASH/ASPC/NMA/ PCNA Guideline for the Prevention, Detection, Evaluation, and Management of High Blood Pressure in Adults. A Report of the American College of Cardiology/American Heart Association Task Force on Clinical Practice Guidelines.

3. Khera R, Lu Y, Saxena A, Nasir K, Krumholz HM (2017) The Impact of 2017 ACC/AHA Guidelines on the Prevalence of Hypertension and Eligibility for Anti-Hypertensive Therapy in the United States and China. bioRxiv

4. Muntner P, Carey RM, Gidding S, Jones DW, Taler SJ, et al. (2017) Potential U.S. Population Impact of the 2017 American College of Cardiology/American Heart Association High Blood Pressure Guideline. Journal of the American College of Cardiology 71: 109-118. 